\title{
Regulatory aspects of esterase 6 activity variation in sibling Drosophila species
}

\author{
JILL KAROTAM* $¥ \ddagger$ \& JOHN G. OAKESHOTT* \\ *CSIRO Division of Entomology, P.O. Box 1700, Canberra, A.C.T. 2601; tDivision of Biochemistry and Molecular \\ Biology, Australian National University, P.O. Box 4, Canberra, A.C.T. 2601, Australia
}

\begin{abstract}
Esterase 6 in Drosophila melanogaster, Drosophila simulans and Drosophila mauritiana is produced in several life stages and diverse tissues, but the major pulse of expression is in the sperm ejaculatory duct of adult males. Comparison of EST6 activity levels among several lines of $D$. melanogaster, $D$. simulans and $D$. mauritiana reveals two major quantitative differences among the species. First, newly eclosed females of both $D$. simulans and $D$. mauritiana show significantly higher EST6 activity than those of $D$. melanogaster. Secondly, 5-day-old adult $D$. simulans have significantly higher activities than $D$. mauritiana in both sexes and significantly higher activity than $D$. melanogaster in males. The genetic bases of the differences between $D$. melanogaster and the other species are investigated through germ line transfer of the D. simulans and D. mauritiana Est- 6 genes plus $1.2 \mathrm{~kb}$ of $5^{\prime}$ and $0.2 \mathrm{~kb}$ of their $3^{\prime}$ flanking sequences into $D$. melanogaster. The newly eclosed female activities of the transformants resemble those of the two donor species, suggesting that the interspecific differences in this aspect of expression are due to cis-inherited factors contained within the transferred DNA. In contrast, the 5-day adult activity of the $D$. simulans transgene resembles the recipient species, D. melanogaster, suggesting that the difference between $D$. simulans and $D$. melanogaster in this aspect of expression is due to trans-acting factors. We also find that third instar larval activities of the $D$. simulans transgene and 5-day male activities of the $D$. mauritiana transgene are lower than those of either parental species, suggesting that not all the promoter elements relevant to these aspects of expression are included in the transferred DNA.
\end{abstract}

Keywords: activity variation, Drosophila, esterase 6, gene regulation, germ line transformation, interspecific comparisons.

\section{Introduction}

Evidence is accumulating that evolutionary change in gene expression can result from two types of regulatory mutation. One involves $c i s$-inherited mutations in the gene's promoter and the other involves trans-inherited mutations in protein(s) that directly or indirectly affect the functioning of the promoter. While analyses of gene expression in interspecific hybrids and interspecific tissue transplants have provided evidence of both types of change (e.g. Aronshtam \& Kuzin, 1974; Cavener, 1985; Kuhn \& Sprey, 1987), a more direct test is now available. Specifically, interspecific gene transfer experiments can test whether species-specific differences in the expression of a particular transgene are due to cis-acting elements within the introduced DNA, or to trans-acting factors in the host genome, or to a combination of both.

‡Correspondence. Present Address: Biology Department, Queen’s University, Kingston, Ontario, Canada, K7L 5N6.
Most gene transfer experiments among Drosophila species have used $D$. melanogaster as a host and several have demonstrated that observed interspecific differences are due to cis-acting elements directing donor species-specific patterns of expression (reviewed in Dickinson, 1991). Relatively few studies have yielded evidence for trans-acting control over interspecific differences in expression ( $D d c$, Bray \& Hirsh, 1986; Adh, Fischer \& Maniatis, 1986 and Wu et al., 1990; urate oxidase, Wallrath \& Friedman, 1991; Gld, Cavener, 1992 and references therein). It is notable that all four systems in which trans-effects have been demonstrated are responsive to juvenile hormone $(\mathrm{JH})$ and/or ecdysone (Riddiford, 1992).

Here we explore the contribution of cis- and transinherited changes to quantitative differences in esterase 6 (Est-6/EST6) expression among three sibling species of Drosophila. The three species are $D$. melanogaster, $D$. simulans and $D$. mauritiana, which all show significantly higher EST6 expression in mature 
adult males than females, due to a major pulse of EST6 production in the anterior sperm ejaculatory duct (Stein et al., 1984; Morton \& Singh, 1985; Uspenskii et al., 1988). Although little else is known about the tissue distribution of EST6 expression in D. mauritiana, the other two species share many other sites of expression, including substantial activity in haemolymph throughout development (Aronshtam \& Kuzin, 1974). In D. melanogaster at least, the control of EST6 expression in ejaculatory ducts and possibly other tissues is modulated by both $\mathrm{JH}$ and ecdysone (Richmond \& Tepper, 1983; Stein et al., 1984). The overall structure of the Est-6 gene and promoter region is conserved among the three species; two large, perfectly conserved regions in the $350 \mathrm{bp}$ immediately $5^{\prime}$ of the gene are thought to be responsible for basal levels of Est-6 expression while numerous nucleotide substitutions and small insertions/deletions over the next $700 \mathrm{bp} 5^{\prime}$ provide the scope for regulatory divergence (Karotam et al., 1993).

In this study we have undertaken a quantitative comparison of EST6 expression in several lines of $D$. melanogaster, $D$. simulans and $D$. mauritiana across three stages of development and both sexes. We have then tested the genetic basis for the observed interspecific differences, using germ line transformation to introduce two $D$. simulans and one $D$. mauritiana Est6 alleles and their flanking regions into the same $D$. melanogaster background.

\section{Materials and methods}

\section{Fly stocks}

The interspecific activity comparisons were based on 10 field-derived isofemale lines and seven other laboratory stocks of unknown origin. The five $D$. melanogaster stocks used were all isofemale lines: mel-1 to mel-4 were derived from Coffs Harbour, Australia by Cooke et al. (1987) and mel-5 (the source of the Est-6 genomic sequence of Collet et al., 1990) was isolated from Indiana, U.S.A. (stock Dm145 of R.C. Richmond, University S. Florida). The seven $D$. simulans lines comprised: five isofemale lines (sim-1 to sim-5), which were isolated from Coffs Harbour, Australia; and two laboratory stocks, sim- 6 , which was the $D$. simulans stock of A.R. Lohe (Case West. University, Ohio) and sim-7 which was the C135.20 stock from the Bowling Green Stock Center. The five $D$. mauritiana stocks comprised: mau- 1 and mau-2, which were isolated from the G72 strain of D. L. Hartl (Washington University, St. Louis); mau-3, which was the G122 strain of D. L. Hartl; mau-4, which was the $D$. mauritiana stock of A. R. Lohe; and mau-5, which was the Cambridge stock of the Bowling Green Stock Center. The D. simulans strains isolated from the Coffs Harbour population were made homozygous for EST6 by repeated sib-mating. These and all other strains mentioned above were confirmed as pure breeding for EST6 allozyme status by high resolution electrophoretic analyses (methods of Cooke et al., 1987).

Strains of $D$. melanogaster used in germ line transformation experiments were $w ; \Delta 2-3(99 \mathrm{~B}), w$; TM3/ TM6B and $w$; EST6 ${ }^{\text {null }}$ (Sheehan et al., 1979; Robertson et al., 1988). All stocks were maintained at $18^{\circ} \mathrm{C}$ on standard yeast-treacle-cornmeal media (Healy et al., 1991).

\section{Biochemical analyses}

EST6 activity was measured on homogenates of wandering third instar larvae (3IL), newly eclosed ( $\leq 2 \mathrm{~h}$ old) adult males (NEO') and females (NEO) and 5-day-old virgin males (5D $0^{\circ}$ ) and females (5D $\%$ ). Separate homogenates from triplicate cultures were assayed for each wild type and transformant strain. All organisms collected for assays were snap frozen in liquid nitrogen and stored at $-80^{\circ} \mathrm{C}$. Homogenates were prepared by the methods of Healy et al. (1991), divided into aliquots and stored at $-20^{\circ} \mathrm{C}$. Two replicates of each homogenate were assayed spectrophotometrically for $\beta$-naphthyl acetate hydrolysing activity in the presence of eserine sulfate and $p$ chloromercuribenzoate acid (Healy et al., 1991), an assay shown to be specific for EST6 in these species (Sheehan et al., 1979; Healy et al., 1991; J. K., M. J. Healy \& J. G. O., unpublished data). Replicate determinations of the protein concentration of each homogenate were obtained by the method of Bradford (1976) using the BioRad protein assay kit II. EST6 specific activities were calculated as $\mu \mathrm{mol} \beta$-naphthol produced per $30 \mathrm{~min}$ per $\mathrm{mg}$ protein and logarithmic transformations of these values were analysed using Genstat 5 Release 2.1 (Lane et al., 1987; Digby et al., 1989).

\section{Germ line transformation}

Three genomic clones of Est-6 which had been sequenced previously (Karotam et al., 1993; J. K., T. M. Boyce \& J. G. O., unpublished data) were used as donor DNA in the transformation experiments. The two $D$. simulans clones both comprised a $3.00 \mathrm{~kb}$ HindIII/ScaI fragment including the $1.68 \mathrm{~kb}$ Est-6 gene, $1.18 \mathrm{~kb}$ of $5^{\prime}$ flanking sequence and $0.14 \mathrm{~kb}$ of $3^{\prime}$ flanking sequence. The $3.10 \mathrm{~kb} H$ indIII/ScaI fragment from $D$. mauritiana was homologous to those from $D$. simulans but slightly larger, due to a 102 bp insertion 
in the $5^{\prime}$ flanking region $1.14 \mathrm{~kb} 5^{\prime}$ of the start of translation (J. K. \& J. G. O., unpublished data). One of the D. simulans clones (hereafter denoted simE6A) had been isolated from the sim-1 stock above but the strains from which the other two clones (simE6B and mauE6) were derived were no longer available. Plasmid DNA was amplified, prepared and digested by methods described in Karotam et al. (1993). All three fragments were subcloned into the BamHI site of the pCaSpeR transformation vector (Robertson et al., 1988) using $B c l$ linkers (BRL) and the methods of Rusche \& Howard-Flanders (1985) and Karotam et al. (1993). All pCaSpeR clones chosen for injection had the Est- 6 insert in the same orientation, such that the Est-6 and white genes would be convergently transcribed.

DNA from these clones was purified through two rounds of $\mathrm{CsCl}_{2}$ gradient centrifugation and then micro-injected into embryos produced by the $w ; \Delta 2$ $3(99 \mathrm{~B}) \times w$; TM3/TM6B cross, as described by Zachar et al. (1987). Transformants were identified by the rescue of the white eye colour and those in which eye colour assorted independently of the third chromosome markers were crossed into an Est- 6 null background by replacement of their third chromosomes with those from the $D$. melanogaster EST6 ${ }^{\text {null }}$ stock. After establishing homozygous stocks, each integrated construct was confirmed as a single copy by Southern blot hybridization (methods as in Karotam et al., 1993) using the D. melanogaster Est-6 genomic clone as a probe (Collet et al., 1990). Four independent transformants of each $D$. simulans subclone (denoted mel $^{\text {simE6A }}$ lines 1 to 4 and mel ${ }^{\text {simE } 6 B}$ lines 1 to 4 ) and five of the D. mauritiana subclone (mel ${ }^{\text {mauE6 }}$ lines 1 to 5 ) were chosen for further analyses. The inserted DNA was inherited on the $\mathrm{X}$ chromosome of mel ${ }^{\mathrm{sim} E 6 \mathrm{~A}}$ lines 3 and 4, mel ${ }^{\text {simE } 6 \mathrm{~B}}$ line 3 and mel ${ }^{\text {mauE6 }}$ lines 1, 2 and 3, and on the autosomes of the remainder.

\section{Results}

The patterns of EST6 expression in the seventeen lines of $D$. simulans, D. mauritiana and D. melanogaster are broadly similar (Fig. 1). In all three species third instar larval activity tends to be lower than that in adults, newly eclosed adults show lower activity than 5-day adults and adult females show lower activity than males. The latter difference is much less pronounced in newly eclosed flies (generally less than two-fold) than 5-day flies (up to 26-fold, depending on the line).

Table 1 summarizes the analysis of the data from the 17 lines and three species for each of the five activity measures. For most measures there is significant variation (up to six-fold) among lines within species (see Fig.

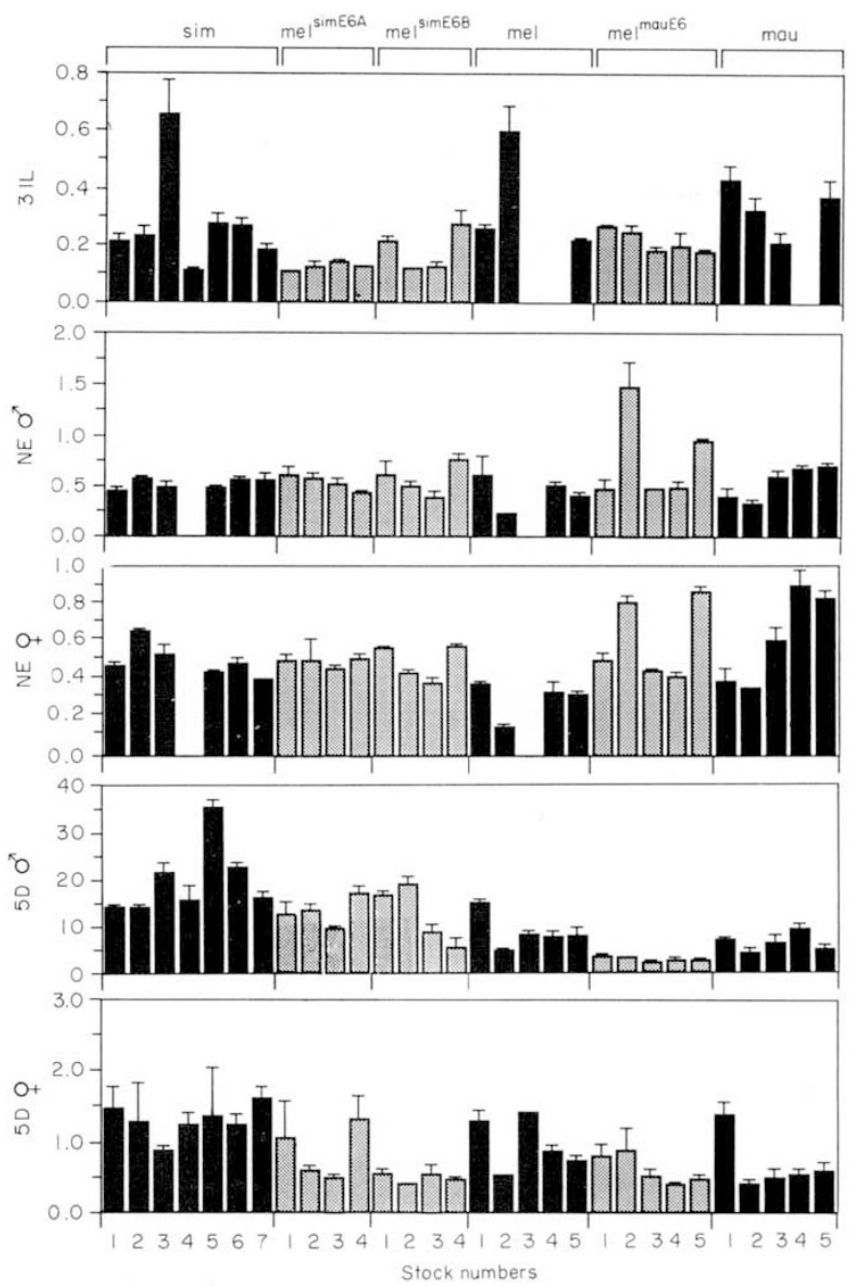

Fig. 1 Mean EST6 specific activities ( \pm S.E.) of wild type $D$. melanogaster (mel), D. simulans (sim) and D. mauritiana (mau) lines (solid bars) and the mel ${ }^{\text {simE6A }}$, mel $^{\text {simE6B }}$ and mel ${ }^{\text {mauE6 }}$ transformant lines (stippled bars) for three developmental stages and both sexes, expressed as $\mu$ mol $\beta$-naphthol produced $/ 30 \mathrm{~min} / \mathrm{mg}$ protein. Note that the mean 5 -day male activity of the five $D$. melanogaster lines was $8.26 \pm 1.70$, significantly higher than the comparable value from the 42 lines tested by Game \& Oakeshott, 1989; $5.27 \pm 0.19$, after adjusting their values to equivalent units. However, our mean 5 -day female activity $(0.95 \pm 0.17)$ was not significantly different to the corresponding (adjusted) value from Game \& Oakeshott, 1989 (0.90 0.03 ). The difference in 5-day male activities suggests that the present study will underestimate interspecific differences involving D. melanogaster, which is as low or lower than either of the other two species for this measure.

1), but two major differences also distinguish the three species, the first of which is in newly eclosed female activities. Pairwise comparisons show this to be due to the relatively low (two-fold lower on average) EST6 activities of $D$. melanogaster females. The second major difference among the species is in 5-day adults, 
Table 1 Analyses of variance ( $F$ ratios with degrees of freedom in brackets) for each of the five EST6 activity measures and the two canonical variates assessing differences among the three species (mel/sim/mau) and among lines within the species (Lines). Significant effects from the three-way comparisons among the species are decomposed into the contributions from the three pairwise comparisons. Note that only cultures for which data were available for all five activity measures were used in the canonical variate analyses

\begin{tabular}{|c|c|c|c|c|c|c|c|}
\hline \multirow{2}{*}{$\begin{array}{l}\text { Source of } \\
\text { variation }\end{array}$} & \multirow{2}{*}{$\begin{array}{l}\text { 3rd instar } \\
\text { larvae }\end{array}$} & \multicolumn{2}{|c|}{ Newly eclosed adults } & \multicolumn{2}{|c|}{ 5-day adults } & \multicolumn{2}{|c|}{ Canonical variates } \\
\hline & & Males & Females & Males & Females & CV1 & $\mathrm{CV} 2$ \\
\hline $\mathrm{mel} / \mathrm{sim} / \mathrm{mau}$ & $\begin{array}{r}0.75 \\
(2,11)\end{array}$ & $\begin{array}{l}1.13 \\
(2,12)\end{array}$ & $\begin{array}{c}5.98^{*} \\
(2,12)\end{array}$ & $\begin{array}{l}17.41^{* * *} \\
(2,14)\end{array}$ & $\begin{array}{c}7.00^{* *} \\
(2,14)\end{array}$ & $\begin{array}{l}16.00^{* *} \\
(2,9)\end{array}$ & $\begin{array}{l}11.98^{* *} \\
(2,9)\end{array}$ \\
\hline Lines & $\begin{array}{l}12.71^{* * *} \\
(11,27)\end{array}$ & $\begin{array}{l}6.21^{* * *} \\
(12,29)\end{array}$ & $\begin{array}{l}19.49^{* * *} \\
(12,27)\end{array}$ & $\begin{array}{l}8.28^{* * *} \\
(14,33)\end{array}$ & $\begin{array}{c}1.91 \\
(14,25)\end{array}$ & $\begin{array}{l}6.53^{* *} \\
(9,8)\end{array}$ & $\begin{array}{l}3.68^{*} \\
(9,8)\end{array}$ \\
\hline $\mathrm{mel} / \mathrm{sim}$ & & & $\begin{array}{l}9.46^{*} \\
(1,8)\end{array}$ & $\begin{array}{l}17.37^{* *} \\
(1,10)\end{array}$ & $\begin{array}{r}3.23 \\
(1,10)\end{array}$ & $\begin{array}{l}6.00^{*} \\
(1,6)\end{array}$ & $\begin{array}{l}18.32^{* *} \\
(1,6)\end{array}$ \\
\hline Lines & $\begin{array}{l}17.12^{* * *} \\
(8,20)\end{array}$ & $\begin{array}{l}4.67^{* * *} \\
(8,19)\end{array}$ & $\begin{array}{l}19.55^{* * * *} \\
(8,18)\end{array}$ & $\begin{array}{l}10.74^{* * *} \\
(10,23)\end{array}$ & & $\begin{array}{l}19.08^{* *} \\
(6,5)\end{array}$ & $\begin{array}{l}3.50 \\
(6,5)\end{array}$ \\
\hline $\mathrm{mel} / \mathrm{mau}$ & & & $\begin{array}{l}7.32^{*} \\
(1,7)\end{array}$ & $\begin{array}{c}0.89 \\
(1,8)\end{array}$ & $\begin{array}{c}2.62 \\
(1,8)\end{array}$ & $\begin{array}{c}0.28 \\
(1,4)\end{array}$ & $\begin{array}{l}29.82^{* *} \\
(1,4)\end{array}$ \\
\hline Lines & $\begin{array}{l}10.13^{* * * *} \\
(5,13)\end{array}$ & $\begin{array}{l}7.80^{* * *} \\
(7,17)\end{array}$ & $\begin{array}{l}22.67^{* * * *} \\
(7,16)\end{array}$ & $\begin{array}{l}6.54^{* * * *} \\
(8,20)\end{array}$ & & $\begin{array}{c}3.27 \\
(4,3)\end{array}$ & $\begin{array}{c}3.03 \\
(4,3)\end{array}$ \\
\hline $\operatorname{sim} / \mathrm{mau}$ & & & $\begin{array}{c}0.89 \\
(1,9)\end{array}$ & $\begin{array}{l}37.31^{* * *} \\
(1,10)\end{array}$ & $\begin{array}{l}12.96^{* *} \\
(1,10)\end{array}$ & $\begin{array}{l}39.66^{* * * *} \\
(1,8)\end{array}$ & $\begin{array}{c}1.70 \\
(1,8)\end{array}$ \\
\hline Lines & $\begin{array}{l}10.47^{* * *} \\
(9,21)\end{array}$ & $\begin{array}{l}5.93^{* * * *} \\
(9,22)\end{array}$ & $\begin{array}{l}16.20^{* * * *} \\
(9,20)\end{array}$ & $\begin{array}{c}8.44^{* * *} \\
(10,23)\end{array}$ & & $\begin{array}{l}4.85^{*} \\
(8,8)\end{array}$ & $\begin{array}{l}4.14^{*} \\
(8,8)\end{array}$ \\
\hline
\end{tabular}

${ }^{*} P<0.05,{ }^{* *} P<0.01,{ }^{* * *} P<0.001$.

where activity in $D$. simulans is significantly higher (two-fold higher on average) than D. mauritiana in both sexes and significantly higher than $D$. melanogaster in males.

Canonical variate analyses (Digby et al., 1989) were carried out on the five activity measures in order to derive two independent composite variables which best represent the differences between the species. The two derived variables are related to the five original variables as follows:

$\mathrm{CV} 1=-0.64 \ln (3 \mathrm{IL})-0.58 \ln (\mathrm{NEO})+0.26 \ln (\mathrm{NE})$ $+2.58 \ln \left(5 \mathrm{D} 0^{\prime}\right)-0.09 \ln (5 \mathrm{D} \%)-7.10$

$\mathrm{CV} 2=-1.14 \ln (3 \mathrm{IL})-3.87 \ln \left(\mathrm{NEO}^{\prime}\right)+5.13 \ln (\mathrm{NE} \%)$ $+0.02 \ln \left(5 \mathrm{D} 0^{\circ}\right)-0.31 \ln (5 \mathrm{D} \%)-0.26$.

CV1 is mainly weighted on 5-day male activity (with a weighting at least four-fold larger than that for any other activity measure) and distinguishes $D$. simulans from both $D$. melanogaster and D. mauritiana but does not separate the latter two species. CV2 shows a strong negative contribution from newly eclosed males and a strong positive one from newly eclosed females (the weightings for both being at least three-fold larger than those for the other measures) and distinguishes $D$. melanogaster from both $D$. mauritiana and $D$. simulans, but does not separate $D$. simulans and $D$. mauritiana. Both canonical variates contribute fairly evenly to the total variation, CV1 accounting for 59 per cent and CV2 for the remainder. Values for the two canonical variates for each line and species are represented graphically in Fig. 2 while Table 1 shows the corresponding analyses of variance.

Like the wild type lines from all three species, the $D$. melanogaster lines transformed with the two $D$. simulans Est-6 genes also show higher activities in adults than in larvae, in 5-day than newly eclosed adults, and in males than females (Fig. 1). Some significant differences were found among lines within the two types of transformant $\left(\mathrm{mel}^{\mathrm{simE6A}}\right.$ and $\left.\mathrm{mel}^{\mathrm{simE} 6 \mathrm{~B}}\right)$ for several activity measures $\left(F_{6,16}=8.27\right.$ for third instar larvae; $F_{6,16}=3.83$ for newly eclosed males; $F_{6,15}=7.67$ for 5-day males, $P<0.05$ in all cases). These differences presumably reflect effects on the expression of the transgenes due to differences in the position of integration, an effect generally observed in Drosophila transformation studies (e.g. Wu et al., 1990; Kirkpatrick \& Martin, 1992). Significantly, however, there were no overall differences between mel ${ }^{\text {simE6A }}$ and mel ${ }^{\text {simE } 6 \mathrm{~B}}$ lines for any activity measure (the largest $F_{1,6}=4.64, P>0.05$ in all cases), so these data have been pooled for all subsequent analyses. Although the 
endogenous activity of the simE6B gene is not known, nucleotide sequence analysis of the flanking regions of this and the simE6A gene revealed only eight single base pair differences and one single base pair insertion/ deletion in the $1.18 \mathrm{~kb}$ of transformed DNA $5^{\prime}$ of the gene, and five single base pair differences in the 0.14 $\mathrm{kb}$ of $3^{\prime}$ untranslated sequence included in the transformed DNA (J. K., T. M. Boyce \& J. G. O., unpublished data), so the two sets of transformants might be expected to show similar activities.

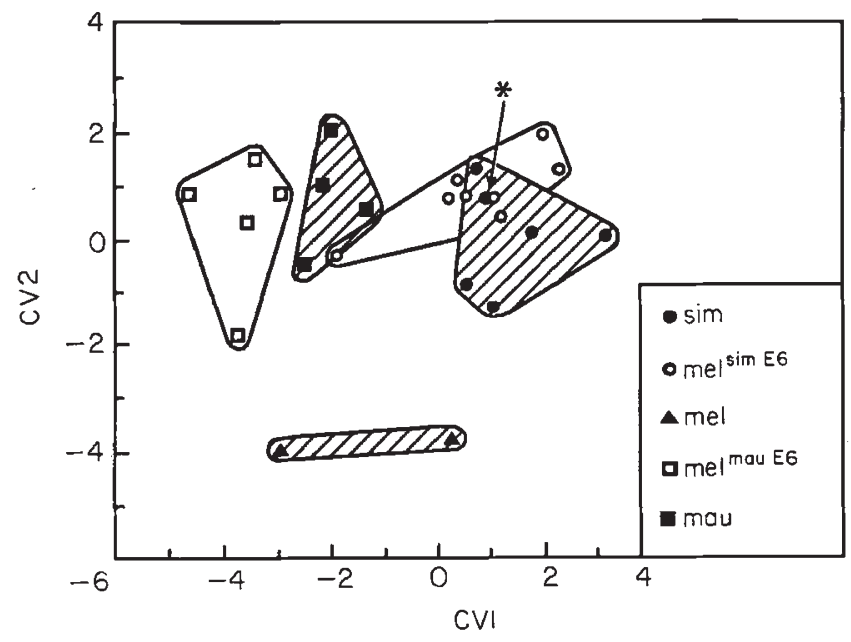

Fig. 2 Distribution of wild type and transformant lines for the two canonical variates CV1 and CV2. Wild type $D$. melanogaster (mel), D. simulans (sim) and D. mauritiana (mau) lines are enclosed in the three shaded regions, the mel ${ }^{\text {simE6 }}$ and melmauE6 transformants in two unshaded regions. The $D$. simulans line ( $\operatorname{sim}-1$ ) from which one of the transgenes was derived is denoted by an asterisk.
The eight mel ${ }^{\text {simE6 }}$ lines differ from the wild type lines of either the donor $(D$. simulans) or recipient $(D$. melanogaster) species in several activity measures (Table 2). The third instar larval activities of the transformants are lower than those of both parental species $(P<0.05$ in both cases $)$. The transformants also differ from $D$. melanogaster $(P<0.01)$ but not $D$. simulans for newly eclosed female activities, although there are no differences from either parental species in newly eclosed male activities. Finally, the transformants do not differ significantly from $D$. melanogaster in 5-day adult male or female activities but their values for both these activities are lower than wild type $D$. simulans ( $P<0.05$ for males, $P<0.001$ for females). Thus for those measures in which the transformants differ from one or the other parental species, the expression of the transgene resembles the donor rather than the recipient species for newly eclosed female activities but resembles the recipient rather than the donor for 5-day adult male and female activities, while for larval activities it differs from both parental species.

The analyses above involve comparison of the mel ${ }^{\text {simE6 }}$ transformants to several lines from both the donor and recipient species. However, one of the lines, sim-1, from which the transgenes had been isolated had been available for inclusion in the activity assays. Therefore the eight transformant lines (the two sets not differing in any activity measure, see above) could be compared with sim-1 for a more specific test of differences from the donor species. (Note that the activities of the transgenes were assayed in a recipient $D$. melanogaster line homozygous for an endogenous EST6 $^{\text {null }}$ allele, so the equivalent comparison of the transformants to the recipient line was not mean-

Table 2 Analyses of variance ( $F$ ratios with degrees of freedom in brackets) for each of the five EST 6 activity measures and the two canonical variates assessing differences between the mel ${ }^{\text {simE6 }}$ transformants and each of the two parental species (mel and sim), as well as differences among lines within these groups (Lines). Note that only cultures for which data were available for all five activity measures were used in the canonical variate analyses

\begin{tabular}{|c|c|c|c|c|c|c|c|}
\hline \multirow{2}{*}{$\begin{array}{l}\text { Source of } \\
\text { variation }\end{array}$} & \multirow{2}{*}{$\begin{array}{l}\text { 3rd instar } \\
\text { larvae }\end{array}$} & \multicolumn{2}{|c|}{ Newly eclosed adults } & \multicolumn{2}{|c|}{ 5-day adults } & \multicolumn{2}{|c|}{ Canonical variates } \\
\hline & & Males & Females & Males & Females & CV1 & $\mathrm{CV} 2$ \\
\hline $\mathrm{mel} / \mathrm{mel}^{\mathrm{simE} 6}$ & $\begin{array}{c}9.54^{*} \\
(1,9)\end{array}$ & $\begin{array}{l}3.25 \\
(1,10)\end{array}$ & $\begin{array}{l}12.74^{* *} \\
(1,10)\end{array}$ & $\begin{array}{l}3.52 \\
(1,11)\end{array}$ & $\begin{array}{r}4.00 \\
(1,11)\end{array}$ & $\begin{array}{l}1.92 \\
(1,8)\end{array}$ & $\begin{array}{l}34.90^{* * * *} \\
(1,8)\end{array}$ \\
\hline Lines & $\begin{array}{l}14.19^{* * * *} \\
(9,22)\end{array}$ & $\begin{array}{l}4.71^{* *} \\
(10,23)\end{array}$ & $\begin{array}{l}8.92^{\text {**** }} \\
(10,23)\end{array}$ & $\begin{array}{l}7.29^{* * * *} \\
(11,25)\end{array}$ & $\begin{array}{c}2.86^{*} \\
(11,21)\end{array}$ & $\begin{array}{l}5.14^{*} \\
(8,8)\end{array}$ & $\begin{array}{c}0.49 \\
(8,8)\end{array}$ \\
\hline $\operatorname{sim} / \mathrm{mel}^{\mathrm{simE} 6}$ & $\begin{array}{c}5.41^{*} \\
(1,13)\end{array}$ & $\begin{array}{l}0.17 \\
(1,12)\end{array}$ & $\begin{array}{r}0.10 \\
(1,12)\end{array}$ & $\begin{array}{l}5.25^{*} \\
(1,13)\end{array}$ & $\begin{array}{l}20.57^{* * * *} \\
(1,13)\end{array}$ & $\begin{array}{l}1.37 \\
(1,12)\end{array}$ & $\begin{array}{r}2.58 \\
(1,12)\end{array}$ \\
\hline Lines & $\begin{array}{l}12.54 * * * \\
(13,30)\end{array}$ & $\begin{array}{l}2.69^{*} \\
(12,28)\end{array}$ & $\begin{array}{c}3.86^{* *} \\
(12,27)\end{array}$ & $\begin{array}{l}7.61^{* * * *} \\
(13,28)\end{array}$ & $\begin{array}{c}1.38 \\
(13,27)\end{array}$ & $\begin{array}{l}5.65^{* * *} \\
(12,13)\end{array}$ & $\begin{array}{c}1.13 \\
(12,13)\end{array}$ \\
\hline
\end{tabular}

${ }^{*} P<0.05,{ }^{* *} P<0.01,{ }^{* * *} P<0.001$. 
ingful.) Only one of the three differences between the transformants and the donor seen in the full analyses above is found to recur in the sim- 1 analysis. This is for 5-day female activities $\left(F_{1,3}=5.08, P<0.05\right.$ on a onetailed test), which had been highly significant $(P<0.001)$ in the full analyses. The less statistically robust differences from $D$. simulans $(P<0.05)$ that the full analyses identified for third instar larvae and 5-day males were not significant in the comparisons to sim- 1 . Thus the relatively large differences from the donor species in 5-day female activity emerge clearly in both analyses, whereas the smaller differences in third instar larval and 5-day male activities are only significant in the full analyses.

Analyses of the EST6 activities of the mel ${ }^{\text {mauE6 }}$ transformants in relation to all the available wild type $D$. melanogaster and $D$. mauritiana lines are shown in Table 3. The transformants have significantly $(P<0.05)$ lower third instar larval activities than wild type $D$. mauritiana but do not differ from $D$. melanogaster. There are no significant activity differences among newly eclosed males and, while the newly eclosed female activities of D. mauritiana and the transformants do not differ, both are significantly lower than those of $D$. melanogaster $(P<0.05)$. The 5-day female activities of the transformants are similar to those of $D$. mauritiana but significantly lower than those of D. melanogaster $(P<0.05)$, whereas the 5-day male activities of the transformants are significantly lower than those of both parental species $(P<0.01$ in both cases). In summary, the transgene carried by the mel $^{\text {mauE} 6}$ transformants resembles the recipient species for third instar larval activities, and the donor species for newly eclosed and 5-day female activities; however their 5-day male activities are significantly lower than those of both parental species.

Since the $D$. mauritiana line from which the donor DNA was isolated was not available, no parallel of the 'sim-1 analyses' above could be carried out for $D$. mauritiana. In its absence we note that the only differences that were significant below $P<0.01$ in the full $D$. mauritiana analyses were the low 5-day male activities of the transformants relative to both parental species.

Comparison of all the transformants to their respective parental species for the canonical variates (Fig. 2, Tables 1-3) shows that for CV2 the transformants resemble the donor species and differ from the recipient. Thus CV2 (with opposing contributions from newly eclosed male and female activities) distinguishes wild type lines of $D$. melanogaster from both $D$. simulans and $D$. mauritiana and the transformants overlap the respective donor species $(D$. simulans and $D$. mauritiana) but are clearly distinguished from the recipient $(D$. melanogaster; $P<0.001$ and $P<0.05$, respectively). Although CV1 (mainly weighted for 5-day male activities) clearly distinguishes the wild type $D$. melanogaster and $D$. simulans lines, the values of CV1 for the mel ${ }^{\operatorname{simE} 6}$ transformants lie between those of both parental species and do not differ significantly from either. CV1 does not show a statistically significant difference between $D$. melanogaster and $D$. mauritiana either, but in this case the mel ${ }^{\text {mauE6 }}$ transformants differ from the donor, D. mauritiana $(P<0.01)$, but not the recipient, D. melanogaster.

The canonical analysis is thus consistent with the univariate analyses in terms of differences from the recipient species. These differences are clearly evident for the transgenes from both species for CV2, which is

Table 3 Analyses of variance ( $F$ ratios with degrees of freedom in brackets) for each of the five EST6 activity measures and the two canonical variates assessing differences between the mel ${ }^{\text {mauE6 }}$ transformants and each of the two parental species (mel and mau), as well as differences among lines within these groups (Lines). Note that only cultures for which data were available for all five activity measures were used in the canonical variate analyses

\begin{tabular}{|c|c|c|c|c|c|c|c|}
\hline \multirow{2}{*}{$\begin{array}{l}\text { Source of } \\
\text { variation }\end{array}$} & \multirow{2}{*}{$\begin{array}{l}\text { 3rd instar } \\
\text { larvae }\end{array}$} & \multicolumn{2}{|c|}{ Newly eclosed adults } & \multicolumn{2}{|c|}{ 5-day adults } & \multicolumn{2}{|c|}{ Canonical variates } \\
\hline & & Males & Females & Males & Females & $\mathrm{CV} 1$ & $\mathrm{CV} 2$ \\
\hline $\mathrm{mel} / \mathrm{mel}^{\mathrm{mau} E G}$ & $\begin{array}{c}2.53 \\
(1,6)\end{array}$ & $\begin{array}{c}2.82 \\
(1,7)\end{array}$ & $\begin{array}{c}8.84^{*} \\
(1,7)\end{array}$ & $\begin{array}{l}23.63^{* *} \\
(1,8)\end{array}$ & $\begin{array}{l}6.87^{*} \\
(1,8)\end{array}$ & $\begin{array}{l}4.90 \\
(1,5)\end{array}$ & $\begin{array}{l}13.22^{*} \\
(1,5)\end{array}$ \\
\hline Lines & $\begin{array}{l}10.54^{* * *} \\
(6,16)\end{array}$ & $\begin{array}{l}12.16^{* * *} \\
(7,17)\end{array}$ & $\begin{array}{l}37.25^{* * *} \\
(7,17)\end{array}$ & $\begin{array}{l}3.88^{* * *} \\
(8,20)\end{array}$ & $\begin{array}{l}2.37 \\
(8,15)\end{array}$ & $\begin{array}{c}2.11 \\
(5,7)\end{array}$ & $\begin{array}{c}2.14 \\
(5,7)\end{array}$ \\
\hline $\mathrm{mau} / \mathrm{mel}^{\mathrm{mauE} 6}$ & $\begin{array}{l}9.22^{*} \\
(1,7)\end{array}$ & $\begin{array}{c}1.09 \\
(1,8)\end{array}$ & $\begin{array}{c}0.00 \\
(1,8)\end{array}$ & $\begin{array}{l}24.82^{* *} \\
(1,8)\end{array}$ & $\begin{array}{l}0.14 \\
(1,8)\end{array}$ & $\begin{array}{l}18.64^{* *} \\
(1,7)\end{array}$ & $\begin{array}{c}0.40 \\
(1,7)\end{array}$ \\
\hline Lines & $\begin{array}{c}3.03^{*} \\
(7,17)\end{array}$ & $\begin{array}{l}14.56^{\text {**** }} \\
(8,20)\end{array}$ & $\begin{array}{l}27.30^{* * * * *} \\
\langle 8,19\rangle\end{array}$ & $\begin{array}{r}2.39 \\
(8,20)\end{array}$ & $\begin{array}{l}3.56^{*} \\
(8,19)\end{array}$ & $\begin{array}{r}0.96 \\
(7,10)\end{array}$ & $\begin{array}{r}2.65 \\
(7,10)\end{array}$ \\
\hline
\end{tabular}

${ }^{*} P<0.05,{ }^{* * *} P<0.01,{ }^{* * *} P<0.001$. 
heavily weighted for the variable (newly eclosed female activity) that showed the clearest differences from the recipient for both donor species in the univariate analyses. On the other hand, the only differences from the donor species revealed by the canonical variate analysis is for the mauE6 transgene for CV1, which is mainly weighted for 5-day male activity. Four other differences from the donor species had also emerged from the univariate analyses, notably for 5-day males and females for $D$. simulans and third instar larvae for both these species, but clearly none of these differences would contribute to the CV1 effect for the mauE6 transgene.

\section{Discussion}

A systematic comparison of EST6 activity levels in $D$. melanogaster, D. simulans and D. mauritiana reveals broadly similar stage- and sex-specific patterns of EST6 expression. Nevertheless, there are also significant intraspecific as well as interspecific differences in EST6 activity levels in the three developmental stages and in both sexes.

Significant activity variation is found among lines within all three species in almost all measures, with up to six-fold variation in individual measures. Thus the levels of intraspecific variation in EST6 activity reported previously for $D$. melanogaster lines (Game \& Oakeshott, 1989) recur among our lines of this species and are also evident in small random samples of $D$. simulans and D. mauritiana. Game \& Oakeshott (1989) found that the variation they detected in EST6 activity among $D$. melanogaster lines reflects differences in the amount of enzyme and that the variation in 4-5-day adult male and female activities is only weakly correlated. This suggests that the variation is due to regulatory rather than structural differences in EST6, and that different regulatory polymorphisms affect activity in the two sexes. This is perhaps not surprising given the differences in the tissue distribution of EST6 between the sexes in these species (Aronshtam \& Kuzin, 1974; Healy et al., 1991). For example, in 5-day adults the majority of male activity is in ejaculatory ducts while in females the majority of activity is in haemolymph (Aronshtam \& Kuzin, 1974; Morton \& Singh, 1985; Uspenskii et al., 1988; Healy et al., 1991).

Physiological and genetic data identify several levels at which EST6 expression is regulated and all are potentially subject to intraspecific polymorphism, either in cis- or trans-acting factors. The physiological data come from transplants of male reproductive organs into female abdomens (Aronshtam \& Kuzin, 1974) and from exposure of dissected abdomens and explants of ejaculatory ducts to $\mathrm{JH}$ and ecdysone
(Richmond \& Tepper, 1983; Stein et al., 1984). These data indicate that both hormones contribute to the induction of EST6 in adults of $D$. melanogaster, although the effect in males may be at least partly mediated by an intermediary signal associated with the accessory glands. Genetic data pertaining to EST6 regulation implicate both cis- and trans-control of activity variation, at least within $D$. melanogaster. Significant activity variation due to trans-acting polymorphisms on the $\mathrm{X}$ chromosome has been described among both laboratory and field derived strains (Aronshtam \& Korochkin, 1975; Richmond \& Tepper, 1983; Tepper et al., 1984), while cis-acting effects are implicated from correlations between restriction fragment length polymorphisms in the Est-6 promoter and activity variation in field derived isochromosomal lines (Game \& Oakeshott, 1990).

The two major interspecific differences in EST6 expression we have found are the relatively low newly eclosed female activities of $D$. melanogaster and the relatively high 5-day adult activities of $D$. simulans, these differences also being reflected in the canonical variate analyses. Although we have no direct causal evidence, we favour regulatory rather than structural differences as an explanation for these interspecific differences. Structural differences in the EST6 protein would be more likely to produce systemic effects on EST6 activity levels rather than the temporal- and sexspecific effects seen here. Further evidence that these differences are not systemic is that reproductive tract activity accounts for differing proportions of total male activity among single lines of these species, being a higher proportion of male activity in $D$. simulans than in either D. melanogaster or D. mauritiana ( 70 vs. 47 and 38 per cent respectively, Morton \& Singh, 1985). (Note however that we find none of the three species express EST6 in testes, whereas Morton \& Singh (1985) reported that $D$. mauritiana, albeit not the other two species, does show EST6 activity in this tissue; J. K. \& J. G. O., unpublished data.)

The aims of our germ line transformation of $D$. melanogaster with Est -6 genes from $D$. simulans and $D$. mauritiana have been to distinguish between cis- and trans-acting effects as the causes of the differences in EST6 expression among the three species. The restriction fragments from $D$. simulans and $D$. mauritiana used in our interspecific gene transfer experiments are precisely homologous to those used previously for similar experiments within $D$. melanogaster (Oakeshott et al., 1990; M. J. Healy, M. M. Dumancic \& J. G. O., unpublished data). In general, the EST6 activities of the interspecific transformants overlap the range of values for at least one parental species in almost all measures, confirming previous evidence from $D$. melanogaster 
that $1.2 \mathrm{~kb}$ of $5^{\prime}$ and $0.2 \mathrm{~kb}$ of $3^{\prime}$ flanking sequences are generally sufficient for most qualitative and quantitative aspects of EST6 expression (Oakeshott et al., 1990; M. J. Healy, M. M. Dumancic \& J. G. O., unpublished data).

However, two exceptions are that third instar larvae of the D. simulans transformants and 5-day males of the $D$. mauritiana transformants show significantly lower activity than either parental species, suggesting that some species-specific promoter elements affecting these aspects of expression may lie outside the flanking sequences included with the transgenes. It is interesting in this respect that the next 350 bp beyond the $5^{\prime}$ boundary of the transgene constructs show a similar level of divergence to that in the adjacent $350 \mathrm{bp}$ within the constructs, at least between $D$. melanogaster and $D$. simulans (J. K., T. M. Boyce and J. G. O., unpublished data).

As is the case for the majority of interspecific transfer experiments involving Drosophila genes (Dickinson, 1991; Cavener, 1992), the expression of $D$. simulans and D. mauritiana EST6 in the D. melanogaster background more closely resembles the donor species pattern than that of the recipient. Donorspecific levels of EST6 expression indicative of cisinherited controls are clearly seen for both the $D$. simulans and D. mauritiana transgenes in newly eclosed female activities and the canonical variate, CV2, which carries strong but opposing weightings from newly eclosed male and female activities. Similar but smaller donor effects are also apparent for the 5-day female activity of the $D$. mauritiana transgene. All these differences from the pattern of expression of the recipient species presumably reflect interspecific differences in promoter sequences that affect the level and/or tissue-specificity of EST6 expression.

Although the overall structure of the promoter region is conserved across the three species, there are many base substitutions and small insertions/deletions among them that could cause these effects (Karotam et al., 1993). The levels of divergence of $D$. melanogaster from the other two species are low $(\leq 2.3$ per cent $)$ in the $350 \mathrm{bp}$ immediately $5^{\prime}$ of the Est -6 coding region but significantly higher in the next $700 \mathrm{bp}$ of the promoter region ( $\geq 9.8$ per cent). The $0.14 \mathrm{~kb}$ of $3^{\prime}$ untranslated sequence included in the transformed constructs are also highly divergent $(\geq 10.1$ per cent, Karotam et al., 1993).

Evidence for predominant cis-control of interspecific differences in EST6 expression was also apparent after germ line transformation of the $D$. pseudoobscura homologue of Est-6 (termed Est $5 B$ ) into D. melanogaster (Brady \& Richmond, 1990). The major pulse of EST5B expression in D. pseudoobscura is in the adult eye, not the ejaculatory duct as in the case of $D$. melanogaster, and it is notable that detectable sequence similarity between the promoter regions of Est- 6 and Est $5 B$ is confined to the first $174 \mathrm{bp} 5^{\prime}$ of the gene (Brady et al., 1990). Although only 450 bp of $5^{\prime}$, but $1.1 \mathrm{~kb}$ of $3^{\prime}$ flanking sequence, were included with the Est $5 B$ transgene, the major pulse of expression occurred in the adult eye of the transformants, therefore resembling the donor species' pattern of expression.

Unlike the D. pseudoobscura Est-6 homologue, we do find some evidence for trans-acting control of the Est-6 transgenes from D. simulans and D. mauritiana. Thus, the mel ${ }^{\text {simE6 }}$ transformants have significantly lower 5-day female activities than the wild type $D$. simulans lines but are not significantly different from $D$. melanogaster wild type. (Note that we cannot distinguish between cis- and trans-effects for 5-day males, since the donor line (sim-1) and recipient species show similar activity levels; see Fig. 1.) There is also some evidence for trans-effects on the mauE6 transgene in third instar larvae and CV1.

Such trans-effects could be artefacts of our experiment if some of the D. simulans Est-6 promoter was omitted from the transformed sequences, element(s) in the omitted region being absent from the wild type $D$. melanogaster promoter but being responsive to transacting factor(s) found in both species. We cannot discount this possibility, but would note that our evidence for trans-acting effects is consistent with evidence from analysis of $D$. melanogaster/D. simulans hybrids which suggests the existence of species-specific trans-acting modifiers of Est-6 expression on the X chromosome (Aronshtam et al., 1975; Tepper et al., 1982). Hybrid males bearing a $D$. simulans $\mathrm{X}$ chromosome show reduced levels of $D$. melanogaster-derived EST6 activity in all tissues and stages examined (albeit most pronounced in the ejaculatory duct), while $D$. simulans-derived EST6 activity (distinguished by its different electrophoretic mobility) is unaffected (Korochkin et al., 1974; Aronshtam \& Korochkin, 1975). Similar but smaller effects of the D. simulansderived $\mathrm{X}$ chromosome are sometimes seen when a $D$. melanogaster $\mathrm{X}$ chromosome is also present in hybrid females, depending on the parental strains used (Korochkin et al., 1974).

It is an intriguing feature of our data that while ciscontrols are clearly evident for the interspecific activity differences in newly eclosed flies (namely the newly eclosed female and $\mathrm{CV} 2$ results for both the $D$. simulans and D. mauritiana transgenes), trans-controls are more in evidence for the 5-day adult activities (namely 5-day female activity for the $D$. simulans transgene and CV1 for the D. mauritiana transgene). Our 
current knowledge of EST6 physiology is insufficient to interpret this pattern in any detail although it is clear that induction by $\mathrm{JH}$ and/or ecdysone contributes to the increase in EST6 activity post-eclosion (Richmond \& Tepper, 1983; Stein et al., 1984). This, together with the fact that both variation in ecdysone titre and the gene for a putative cytosolic $\mathrm{JH}$ binding protein map to the $\mathrm{X}$ chromosome in D. melanogaster (Kiss et al., 1978; Klose et al., 1980; Shemshedini \& Wilson, 1990 ), suggests that these hormones may be involved in the interspecific trans-effects on EST6 expression documented herein. For ecdysone at least, specific promoter elements that respond to the hormone have now been identified in a number of genes (Andres \& Thummel, 1992) and we have identified three putative consensus ecdysone response elements $(\mathrm{AG}(\mathrm{C} / \mathrm{T}) \mathrm{G}(\mathrm{C})$ T)A; Pongs, 1988) in the $1.18 \mathrm{~kb}$ of $5^{\prime}$ Est-6 flanking sequences in all three species (starting at positions $-269,-443$ and -662 in the $D$. melanogaster sequence; Karotam et al., 1993). Some ecdysoneinducible genes (e.g. P1, Maschat et al., 1991) are tissue-specific in their response to the hormone and may act as tissue-specific transcription factors (Andres \& Thummel, 1992).

Four other Drosophila genes for which interspecific germ line transfer experiments have previously shown some trans-control of interspecific expression differences ( $D d c$, $A d h$, urate oxidase and $G l d$, see Introduction) are all also responsive to changing $\mathrm{JH}$ and/or ecdysone titres (Riddiford, 1992, and references therein). This emerging pattern suggests that while cisacting changes will be powerful agents for evolutionary changes in the expression of individual genes, evolutionary changes in the titres of key hormones could affect the expression of whole suites of genes.

\section{Acknowledgements}

We would like to thank Marion Healy for her advice on transformation techniques and valuable comments on the manuscript, and Thomas Boyce, Peter Cooke, Anne Game, Mark Myers, Wendy Odgers, Rollin Richmond, Robyn Russell, Lis van Papenrecht and Bill Vogt for many helpful discussions. We would also like to thank Marion Healy and Mira Dumancic for sharing unpublished data.

\section{References}

ANDRES, A. J. AND THUMMEL, C. S. 1992. Hormones, puffs and flies: the molecular control of metamorphosis by ecdysone. Trends Genet., 8, 132-138.

ARONSHTAM, A. A. AND KOROCHKIN, L. 1. 1975. Expression of allelomorphs of the locus esterase 6 in hybrids between
Drosophila melanogaster and Drosophila simulans. Genetika, 11, 87-91.

ARONSHTAM, A. A. AND KUZIN, B. A. 1974. Investigation of the activity of esterase 6 in ontogenesis of Drosophila melanogaster and Drosophila simulans. Zh. Obshch. Biol., 35, 926-933.

ARONSHTAM, A. A., KUZIN, B. A. AND KOROCHKIN, L. 1. 1975. Features of the phenotypic expression of alleles of the esterase 6 locus in hybrids between Drosophila melanogaster and Drosophila simulans. Genetika, 11, 92-96.

BRADFORD, M. M. 1976. A rapid and sensitive method for the quantitation of microgram quantities of protein utilising the principle of protein-dye binding. Analyt. Biochem., 72, 248-254.

BRADY, J. P. AND RICHMOND, R. C. 1990. Molecular analysis of evolutionary changes in the expression of Drosophila esterases. Proc. Natl. Acad. Sci. U.S.A., 87, 8217-8221.

BRADY, J. P., RICHMOND, R. C. AND OAKESHOTT, J. G. 1990. Cloning of the esterase 5 locus from Drosophila pseudoobscura and comparison with its homologue in D. melanogaster. Mol. Biol. Evol., 7, 525-546.

BRAY, S. J. AND HIRSH, J. 1986. The Drosophila virilis dopa decarboxylase gene is developmentally regulated when integrated into Drosophila melanogaster. EMBO J., 5, 2305-2311.

CAVENER, D. R. 1985. Co-evolution of the glucose dehydrogenase gene and the ejaculatory duct in the genus Drosophila. Mol. Biol. Evol., 7, 141-149.

CAVENER, D. R. 1992. Transgenic animal studies on the evolution of regulatory circuitries. BioEssays, 14, 237-244.

COLLET, C., NIELSEN, K. M., RUSSELL, R. J. KARL, M., OAKESHOTT, J. G. AND RICHMOND, R. C. 1990. Molecular analysis of duplicated esterase genes in Drosophila melanogaster. Mol. Biol. Evol., 7, 9-28.

COOKE, P. H., RICHMOND, R. C. AND OAKESHOTT, J. G. 1987. High resolution electrophoretic variation at the esterase 6 locus in a natural population of Drosophila melanogaster. Heredity, 59, 259-264.

DiCKınson, w. J. 1991. The evolution of regulatory genes and patterns in Drosophila. In: Hecht, M. K., Wallace, B. and MacIntyre, R. J. (eds) Evolutionary Biology, vol. 25, Plenum Press, New York, pp. 127-173.

DigBy, P., Galwey, N. ANd Lane, P. 1989. Genstat 5 A Second Course. Clarendon Press, Oxford.

FisCheR, J. A. AND MANiAT1S, T. 1986. Regulatory elements involved in Drosophila Adh gene expression are conserved in divergent species and separate elements mediate expression in different tissues. EMBO J., 5, 1275-1289.

GAME, A. Y. AND OAKESHOTT, J. G. 1989. Variation in the amount and activity of esterase 6 in a natural population of Drosophila melanogaster. Heredity, 62, 27-34.

GAME, A. Y. AND OAKESHOTT, J. G. 1990. Associations between restriction site polymorphism and enzyme activity variation for esterase 6 in Drosophila melanogaster. Genetics, 126, 1021-1031.

HEALY, M. J., DUMANCIC, M. M. AND OAKESHOTT, J. G. 1991. Biochemical and physiological studies of soluble esterases from Drosophila melanogaster. Biochem. Genet., 29, 365-388. 
KAROTAM, J., DELVES, A. C. AND OAKEShoTT, J. G. 1993. Conservation and change in structural and $5^{\prime}$ flanking sequences of esterase 6 in sibling Drosophila species. Genetika, in press.

KIRKPATRICK, R. B. AND MARTIN, P. F. 1992. Tissue-specific effects on alcohol dehydrogenase expression in Drosophila melanogaster. Mol. Gen. Genet., 232, 135-144.

KISS, I., SZABAD, J. AND MAJOR, J. 1978. Genetic and developmental analysis of puparium formation in Drosophila. Mol. Gen. Genet., 164, 77-83.

KLOSE, W., GATEFF, E., EMMERICH, H. AND BEIKIRCH, H. 1980. Developmental studies on two ecdysone-deficient mutants of Drosophila melanogaster. Wilhelm Roux Arch., 189, $57-62$.

KOROCHKIN, L. I., ARONSHTAM, A. A. AND MATVEEVA, N. M. 1974. Genetics of esterases in Drosophila. III. Influence of different chromosomes on esterase pattern in Drosophila. Biochem. Genet., 12, 9-24.

KUHN, D. T. AND SPREY, Th. E. 1987. Regulation of NADP-Malic enzyme in the eye-antennal disc of $D$. melanogaster $/ D$. simulans hybrids: Evidence for cis- and trans-regulation. Genetics, 115, 277-281.

LANE, P., GALWEY, N. AND ALVEY, N. 1987. Genstat 5 An Introduction. Clarendon Press, Oxford.

MASCHAT, F., DUBERTRET, M.-L. AND LEPESANT, J.-A. 1991. Transformation mapping of the regulatory elements of the ecdysone-inducible $P 1$ gene in Drosophila melanogaster. Mol. Cell. Biol., 11, 2913-2917.

MORTON, R. A. AND SINGH, R. S. 1985. Biochemical properties, homology and genetic variation of the Drosophila 'nonspecific' esterases. Biochem. Genet., 23, 959-973.

OAKESHOTT, J. G., HEALY, M. J. AND GAME, A. Y. 1990. Regulatory evolution of $\beta$-carboxyl esterases in Drosophila. In: Barker, J. S. F., Starmer, T. W. and MacIntyre, R. J. (eds) Ecological and Evolutionary Genetics of Drosophila, Plenum Press, New York, pp. 359-387.

PONGS, o. 1988. Ecdysteroid-regulated gene expression in Drosophila melanogaster. Eur. J. Biochem., 175, 199-204.

RICHMOND, R. C. AND TEPPER, C. S. 1983. Genetic and hormonal regulation of esterase 6 activity in male Drosophila melanogaster. In: Rattazzi, M. C., Scandalios, J. G. and Whitt, G. S. (eds) Isozymes: Current Topics in Biological and Medical Research, vol. 9, A. R. Liss, New York, pp. 91-106.

RIDDIFORD, L. M. 1992. Hormones and Drosophila development. In: Bate, M. and Martinez-Arias, A. (eds) The Development of Drosophila. Cold Spring Harbor Laboratory Press, New York, in press.
ROBERTSON, H. M., PRESTON, C. R., PHILLIS, R. W., JOHNSON-SCHLITZ, D. M., BENZ, W. K. AND ENGELS, W. R. 1988. A stable genomic source of $P$ element transposase in Drosophila melanogaster. Genetics, 118, 461-470.

RUSCHE, J. R. AND HOWARD-FLANDERS, P. 1985. Hexamine cobalt chloride promotes intermolecular ligation of blunt end DNA fragments by T4 DNA ligase. Nucl. Acids Res., 13, 1997-2008.

SHEEHAN, K., RICHMOND, R. C. AND COChRANE, B. J. 1979. Studies of esterase 6 in Drosophila melanogaster. III. The developmental pattern and tissue distribution. Insect Biochem., 9, 443-450.

SHEMSHEDINI, L. AND WILSON, T. G. 1990. Resistance to juvenile hormone and an insect growth regulator in Drosophila is associated with an altered cytosolic juvenile hormonebinding protein. Proc. Natl. Acad. Sci. U.S.A., 87, 2072-2076.

STEIN, S. P., TEPPER, C. S., ABLE, N. D. AND RICHMOND, R. C. 1984. Studies of esterase 6 in Drosophila melanogaster. XVI. Synthesis occurs in the male reproductive tract (anterior ejaculatory duct) and is modulated by juvenile hormone. Insect Biochem., 14, 527-532.

TEPPER, C. S., RICHMOND, R. C., TERRY, A. L. AND SENIOR, A. 1982. Studies of esterase 6 in Drosophila melanogaster. XI. Modification of esterase 6 activity by unlinked genes. Genet. Res., 40, 109-125.

TEPPER, C. S., TERRY, A. L., HOLMES, J. E. AND RICHMOND, R. C. 1984. Studies of esterase 6 in Drosophila melanogaster. XIV. Variation of esterase 6 levels controlled by unlinked genes in natural populations. Genet. Res., 43, 181-190.

USPENSKII, I. I., LUDVIG, M. Z. AND KOROCHKIN, L. I. 1988. Comparative analysis of carboxylesterases in different organs of male Drosophila reproductive system in the melanogaster subgroup. Zh. Obshch. Biol., 49,601-610.

WALLRATH, L. L. AND FRIEDMAN, T. B. 1991. Species differences in the temporal pattern of Drosophila urate oxidase gene expression are attributed to trans-acting regulatory changes. Proc. Natl. Acad. Sci. U.S.A., 88, 5489-5493.

WU, C.-Y., MOTE, J. AND BRENNAN, M. D. 1990. Tissue-specific expression phenotypes of Hawaiian Drosophila $A d h$ genes in Drosophila melanogaster transformants. Genetics, 125, 599-610.

ZACHAR, Z., GARZA, D., CHOU, T.-B., GOLAND, J. AND BINGHAM, P. M. 1987. Molecular cloning and genetic analysis of the suppressor-of-white-apricot locus from Drosophila melanogaster. Mol. Cell. Biol., 7, 2498-2505. 